\title{
El storytelling de la \\ campaña presidencial portuguesa de 2016. Rebelo de Sousa vs. Sampaio da Nóvoa
}

Jaime López Díez*
Jorge Verissimo**

Resumen: El objetivo de este estudio ha sido analizar el storytelling de la campaña política de los dos principales candidatos a la presidencia de Portugal en las elecciones de 2016: Marcelo Rebelo de Sousa y António Sampaio da Nóvoa. La metodología empleada ha sido el análisis de contenido de los Personajes y la Trama en sus discursos de presentación de candidatura y en las noticias sobre sus campañas en los periódicos Expresso y Público. Los resultados muestran, en el análisis de prensa escrita, una relación entre el uso del personaje del villano y las encuestas de intención de voto, una mayor referencia en ambos candidatos a la situación de equilibrio final, así como que Rebelo de Sousa basó su narrativa en el héroe, mientras que en Sampaio da Nóvoa es significativa el recurso al villano.

Palabras clave: Storytelling. Narrativa. Campaña política. Portugal. Personajes. Trama.

\section{The storytelling of the 2016 Portuguese presidential campaign. Rebelo de Sousa vs. Sampaio da Nóvoa}

Abstract: The objective of this study is to analyse the storytelling of the political campaigns of the main candidates in the 2016 Portuguese presidential campaign: Marcelo Rebelo de Sousa and António Sampaio da Nóvoa. The Methodology followed has been a content analysis of Characters and Plot in their announcement speeches, and news about their campaigns in newspapers Expresso and Público. The Results show that, in the analysis of newspapers, there is a relationship between the use of the villain and the election polls; the final state of equilibrium was the most used plot point by both candidates, as well as that Rebelo de Sousa's narrative was based on the hero, while in Sampaio da Nóvoa's the villain is a significant category.

Keywords: Storytelling. Narrative. Political campaign. Portugal. Characters. Plot.
Recebido: 18.11 .19 Aprovado: 02.09.20

\author{
* Jaime López Díez é \\ professor associado \\ do Departamento \\ de Ciências da \\ Comunicação \\ Aplicada da \\ Universidad \\ Complutense de \\ Madrid (UCM), \\ Madrid, Espanha. \\ Orcid: 0000- \\ 0003-3443-5910. \\ <jailop05@ucm.es>. \\ ** Jorge Verissimo \\ é doutor em \\ sociologia, na \\ especialidade \\ de sociologia da \\ comunicação, \\ pelo Instituto \\ Universitário de \\ Lisboa (2005). \\ Professor \\ coordenador na \\ Escola Superior de \\ Comunicação Social \\ (ESCS), do Instituto \\ Politécnico de \\ Lisboa (IPL), diretor \\ da licenciatura \\ em Publicidade e \\ Marketing. Lisboa, \\ Portugal. \\ Orcid: 0000 \\ 0001-8045-4114. \\ <jverissimo@escs. \\ ipl.pt>.
}

Introducción

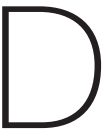
istintos autores han señalado la necesidad de estudiar con mayor profundidad las historias que se emplean en política y en movimientos sociales (Polletta, 2006; Swerts, 2015), así como que estudios multidisciplinares aborden las narrativas políticas (Björninen, Hatavara y Mäkelä, 2020; Jasper, Young 
1. La última revisión de la Constitución de la República Portuguesa tuvo lugar en 2005.

2. Además del Presidente y el Gobierno, los otros dos órganos de soberanía de Portugal son la Asamblea de la República y los Tribunales (art. 110.1).

3. Oficialmente registrado como PPD/PSD, ya que fue fundado como Partido Popular Democrático (PPD) en 1974, y en 1976, pasó a llamarse Partido Social Demócrata (PSD).

\section{El primer} presidente constitucional pertenecía al Partido Renovador Democrático. y Zuern, 2018). El objetivo de este estudio es contribuir a este campo de investigación centrándonos en la trama y los personajes del storytelling político desde un punto de vista narratológico. Para ello, hemos analizado el storytelling (narrativa) de la campaña presidencial portuguesa de 2016, acotando el objeto de estudio a los dos candidatos que obtuvieron un mayor número de votos: Marcelo Rebelo de Sousa y António Sampaio da Nóvoa. El primero fue elegido, a la postre, Presidente de la República de Portugal.

En primer lugar, explicaremos el contexto político y electoral portugués. Luego expondremos una revisión de la literatura académica sobre el storytelling político, centrándonos en la trama y los personajes. A continuación indicaremos los objetivos, preguntas de investigación e hipótesis, así como la metodología seguida. Tras ello, analizaremos los datos obtenidos, y terminaremos con unas conclusiones.

\section{Estructura política portuguesa y contexto de la campaña}

\section{El sistema político portugués}

De acuerdo con la Constitución de la República Portuguesa $(1976 / 2005)^{1}$, el Presidente "representa a la República portuguesa, garantiza la independencia nacional, la unidad del Estado y el regular funcionamiento de las instituciones democráticas y es comandante supremo de las Fuerzas Armadas" (art. 120). Su mandato tiene una duración de cinco años (art. 128). Respecto al sistema electoral, el artículo 126 establece que "será elegido Presidente de la República el candidato que obtuviera más de la mitad de los votos válidamente emitidos"; "si ninguno de los candidatos obtuviera ese número de votos, se procederá a una segunda votación antes del vigésimo primer día siguiente a la primera votación"; y "en esta [segunda] votación concurrirán solo los dos candidatos más votados".

Por su parte, el gobierno lo encabeza el Primer Ministro, a quien compete "dirigir la política general del Gobierno coordinando y orientando la acción de todos los Ministros"; y dirigir el funcionamiento del Gobierno ${ }^{2}$ y sus relaciones de carácter general con los demás órganos del Estado" (art. 201).

Desde la Constitución de 1976, dos partidos políticos han dominado el panorama político portugués: el Partido Socialista (PS) y el Partido Social Demócrata (PSD) ${ }^{3}$. De los cuatro presidentes anteriores a Marcelo Rebelo de Sousa, dos habían pertenecido al PS y uno al PSD ${ }^{4}$. Asimismo, en 20 de los 23 gobiernos constitucionales, el primer ministro ha pertenecido a uno de estos dos partidos: al PS, diez veces, una de ellas 
en coalición con el PSD; y, al PSD, diez veces, tres de ellas en solitario y siete veces en coalición, generalmente con el Centro Democrático Social-Partido Popular (CDS-PP).

\section{La campaña presidencial portuguesa de 2016}

El 24 de enero de 2016 se celebraron las novenas elecciones presidenciales a la República de Portugal desde la Revolución de los Claveles. El presidente saliente, Aníbal Cavaco Silva, es un líder destacado del PSD y también había sido primer ministro. Las elecciones tuvieron lugar durante el gobierno de António Costa, primer ministro y secretario del PS. Su antecesor como primer ministro había sido Pedro Passos Coelho (PSD), quien afrontó la crisis económica e implementó las medidas de austeridad para contener la misma. La campaña electoral oficial tuvo lugar entre el 10 y el 22 de enero de 2016, pero, en los medios de comunicación, había comenzado ya en noviembre de 2015 .

Se presentaron diez candidatos: Marcelo Rebelo de Sousa (Partido Social Democrata - PSD), António Sampaio da Nóvoa (Independiente con el apoyo de una facción del Partido Socialista - PS), Marisa Matias (Bloco de Esquerda - BE), Maria de Belém Roseira (Independiente con el apoyo de una facción del Partido Socialista), Edgar Silva (Partido Comunista - PC), Vitorino Silva (Independiente), Paulo de Morais (Independiente), Henrique Neto (Independiente), Jorge Sequeira (Independiente) y Cándido Ferreira (Independiente). Obtuvo la victoria Marcelo Rebelo de Sousa, con 2.413.956 votos, un 52\% del total, seguido de António Sampaio da Nóvoa, con 1.062.138 votos (22,88\%), y de Marisa Matias, con 469.814 votos (10,12\%). En el presente estudio nos centraremos en los dos candidatos que obtuvieron un mayor número de votos: Marcelo Rebelo de Sousa (en adelante, MRS) y Sampaio da Nóvoa (en adelante, ASN).

ASN anunció su candidatura el 29 de abril de 2015; MRS, el 9 de octubre de 2015. Sus candidaturas se formalizaron el 22 y el 23 de diciembre, respectivamente. Ambos se presentaron como candidatos independientes. No obstante, la candidatura de MRS fue apoyada por el PSD y por el CDS-PP; y la candidatura de ASN, por varios miembros del PS, entre los que destacan el presidente del PS, Cárlos César, y el ex-presidente y ex-primer ministro, Mario Soares. Asimismo, fue recomendada por António Costa, quien también recomendó la candidatura de Maria de Belém.

\section{Marcelo Rebelo de Sousa y António Sampaio da Nóvoa}

Marcelo Rebelo de Sousa (1948) es una persona multifacética, con amplia y reconocida experiencia como político, profesor universitario y periodista. De su extensa actividad política cabe destacar que ha sido presidente del PSD entre 1996 y 1999, y 
ha pertenecido a este partido desde 1974. Participó en la elaboración de la Constitución de la República Portuguesa de 1976. Fue Secretario de Estado durante la VIII legislatura y vice-presidente del Partido Popular Europeo entre 1997 y 1999. Es catedrático de Ciencias Jurídico-Políticas de la facultad de Derecho de la Universidad de Lisboa desde 1990, y especialista en Derecho Constitucional. Como periodista, desde 1973 trabajó para el periódico Expresso, y fue su director entre 1979 y 1981. Un aspecto fundamental para comprender su éxito de la campaña presidencial de 2016 es su popularidad como comentador político en la televisión portuguesa desde los años 90 y, especialmente, desde 2000, cuando fue contratado por la cadena TV1 para su programa el Jornal Nacional. Según Figueiras (2019: 8), allí desarrolló "una nueva forma de hacer comentarios [políticos]" por su "lenguaje accesible", "un discurso pedagógico" y "un componente de entretenimiento en su comentario", con lo que "consiguió comunicarse con los más variados públicos y ser relevante para todos ellos".

Por su parte, António Sampaio da Nóvoa (1954) era poco conocido por los votantes, ya que, hasta las elecciones presidenciales, se había centrado en su carrera académica. Es catedrático de la Universidad de Lisboa, la más prestigiosa de Portugal, y en la que coincidió con MRS. Ejerce la docencia en el Instituto de Educación de dicha universidad y está considerado uno de los mayores especialistas internacionales en Historia de la Educación y Educación comparada (Boto, 2018). Fue rector de la Universidad de Lisboa entre 2006 y 2009 y, desde 2014, es rector honorífico. Su actividad política antes de las elecciones era casi anecdótica. Destaca su asociación a la Liga de Unidade e Ação Revolucionária (Luar) tras la Revolución de los Claveles, así como su participación, en 1976, en las elecciones autárquicas, en las que se eligieron los órganos locales tras la revolución.

\section{Encuestas}

En las elecciones presidenciales portuguesas de 2016, MRS siempre encabezó las encuestas de intención de voto, como muestra la Tabla 1.

Sin embargo, no siempre ASN fue el segundo candidato en intención de voto, ya que Maria de Belém, la otra candidatura recomendada por António Costa, estuvo por delante de ASN en dos encuestas de 2015, y se mantuvo próxima a ASN hasta la encuesta del 17 de enero de 2016. 
TABLA 1

ENCUESTAS DE INTENCIÓN DE VOTO

DE NOVIEMBRE DE 2015 A ENERO DE 2016

\begin{tabular}{|l|c|c|c|c|c|c|c|c|c|}
\hline Instituto & Fecha & Muestra & MRS & ASN & Belém & Edgar Silva & Matias & Neto & Morais \\
\hline Aximage & 20 -ene-16 & 1301 & 51,5 & 22,6 & 9,0 & 5,2 & 6,6 & 0,8 & 2,3 \\
\hline $\begin{array}{l}\text { Univ. Ca- } \\
\text { tólica }\end{array}$ & 17 -ene-16 & 3340 & 52,0 & 22,0 & 8,0 & 3,0 & 8,0 & 1,0 & 3,0 \\
\hline $\begin{array}{l}\text { Euroson- } \\
\text { dagem }\end{array}$ & 13 -ene-16 & 1516 & 54,8 & 16,8 & 16,3 & 4,0 & 4,8 & 1,1 & 1,6 \\
\hline Aximage & 05 -ene-16 & 602 & 52,9 & 16,9 & 11,8 & 2,9 & 2,8 & 1,0 & 2,9 \\
\hline $\begin{array}{l}\text { Euroson- } \\
\text { dagem }\end{array}$ & 21 -dic-15 & 1515 & 52,9 & 16,9 & 11,8 & 4,7 & 4,8 & 1,3 & 1,7 \\
\hline $\begin{array}{l}\text { Univ. Ca- } \\
\text { tólica }\end{array}$ & 06 -dic-15 & 1183 & 62,0 & 15,0 & 14,0 & 3,0 & 3,0 & 1,0 & 1,0 \\
\hline Aximage & 02 -dic-15 & 603 & 54,6 & 13,2 & 13,4 & 2,2 & 2,8 & 0,5 & 1,3 \\
\hline $\begin{array}{l}\text { Euroson- } \\
\text { dagem }\end{array}$ & 18 -nov-15 & 1510 & 48,0 & 16,7 & 18,9 & 5,2 & 6,9 & 2,2 & 1,1 \\
\hline Aximage & 04 -nov-15 & 603 & 56,9 & 15,3 & 13,1 & 2,4 & 0 & 0 & 2 \\
\hline
\end{tabular}

Fuente: elaboración propia, a partir de Pereira (2016).

\section{Marco teórico}

\section{El storytelling político}

El storytelling de las campañas políticas constituye actualmente uno de los factores más relevantes de las mismas (Bergstrand y Jasper, 2018; Jasper, Young y Zuern, 2020; Merelman, 1969; Polletta, 2006). La razón es que el mensaje basado en una historia - entendida esta como una estructura dramática y no como una narración, definida esta última como una secuencia temporal de acontecimientos - es considerado una buena estrategia persuasiva en comparación con el uso de argumentos racionales (Chou, Bleiker, y Premaratna, 2016; McBath y Fisher, 1969: Merelman, 1969; Nimmo, 1985). Una de sus ventajas es que evita o reduce la contra argumentación (Deighton, Romer y McQueen, 1989). Ello convierte a las historias en una estrategia especialmente eficaz cuando el público objetivo sostiene una idea contraria a la del emisor. Otras ventaja es que "la experiencia subjetiva no es necesaria que sea reducida a palabras, por lo que depende de la credibilidad del narrador para comunicarla" (Deighton, Romer y McQueen, 1989: 336).

La literatura académica sobre el estudio del storytelling político es, sin embargo, muy diversa en cuanto a las metodologías utilizadas. Así, entre otras perspectivas, se han analizado distintos niveles de posicionamiento de las narrativas personales empleadas por los políticos (Björninen, Hatavara y Mäkelä, 2020; Jensen, 1980, citado por 
Nimmo, 1985: 33), estudió el storytelling en las campañas políticas estadounidenses desde el punto de vista de su estilo (militar, anunciante, misionero, cruzada y contracruzada); Vromen y Coleman (2013) han empleado cuatro indicadores (identificación personal con la temática; grado de sentimiento compartido por la comunidad; urgencia moral de la campaña; y apoyo de los ciudadanos y de los políticos) para estudiar las campañas de la organización australiana Get Up!; Shanahan, McBeth y Hathaway (2011: 375) han propuesto el Marco Político Narrativo (Narrative Policy Framework, NPF), que ha sido utilizada en diversos escenarios políticos (Gray y Jones, 2016; Kirkpatrick y Stoutenborough, 2018; McBeth y Shanahan, 2004; McMorris, Zanocco y Jones, 2018); y otros autores ha analizado la negatividad o positividad de las campañas políticas (Ansolabehere y lyengar, 1995; Barton, Castillo y Petrie, 2016; Castromil, 2012; Curini, 2017, Lau, Sigelman y Rovner, 2007), lo que implícitamente supone estudiar su storytelling.

Desde el punto de vista de la narratología, el estudio de los discursos narrativos comprende el análisis de (Bal, 1985; Chatman, 1978):

i. un contexto, que implica un espacio y un tiempo;

ii. una trama; y

iii. unos personajes.

En el presente estudio se ha adoptado prioritariamente una perspectiva narratológica para analizar los discursos políticos y, teniendo en cuenta el contexto de la campaña presidencial de 2016, nos hemos centrado en dos de los aspectos narrativos: la trama y los personajes.

\section{La trama en el storytelling político}

Desde la narratología, la trama se define como la organización de los acontecimientos de la historia (Chatman, 1978; Polkinghorne, 1988).

La estructura de la trama de las narrativas políticas se ha abordado de distintas formas. Algunos autores aluden, simplemente, a la estructura de principio, medio y fin, propuesta por Aristóteles en su Poética (1946). Es el caso del NPF propuesto McBeth, Shanahan y Jones (2005: 414-415), quienes, sin embargo, sugieren como útil la estructura descrita por Deborah Stone (2002): empeoramiento (de la historia); progreso con obstáculos; fase de desamparo y fase de control. También se ha estudiado la narrativa según su extensión y el número de temáticas abordadas, como hace Alexander (2010) en su análisis del storytelling digital de Barack Obama; 
o utilizando dos marcadores (actividad y objetos), como hacen Page y Duffy (2016) en su estudio sobre la credibilidad de los políticos en las campañas primarias estadounidenses republicanas de 2008.

Por otro lado, algunos autores se han centrado en un punto concreto de la trama como la resolución (Klein y Mason, 2016); o el clímax, no en el conjunto de la campaña sino como remate de un discurso concreto, como hace Wolf (2017) en relación con los discursos de Hillary Clinton y Donald Trump en las elecciones presidenciales de EE.UU. de 2016.

Desde la narratología, como muestra la Figura 1, se ha considerado que la estructura de la trama de una historia mínima es la siguiente (Todorov, 1963): un personaje principal o protagonista (a través del cual se va a focalizar la historia) se encuentra en una situación de equilibrio inicial que es alterada por un conflicto. El protagonista se enfrenta, así, a una serie de acontecimientos en su camino por resolver el conflicto. En el clímax, típicamente, protagonista y antagonista se enfrentan; el conflicto se resuelve; y ello da lugar a una situación de equilibrio final, favorable o desfavorable para el protagonista, pero en la que este ha cambiado respecto a su situación de equilibrio inicial.

FIGURA 1

ESTRUCTURA DE TRAMA DE LA HISTORIA MÍNIMA

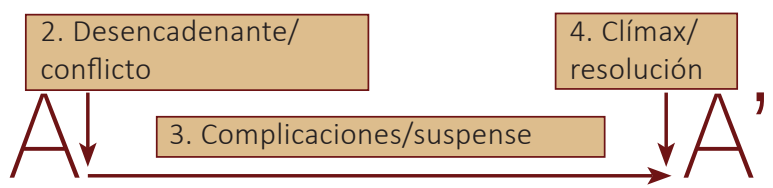

1. Equilibrio inicial

5. Equilibrio final

Fuente: elaboración propia, adaptada de Todorov (1963).

De esta estructura general puede estar ausente alguna de las partes. Por ejemplo, si se omite la situación de equilibrio inicial y el conflicto, nos encontramos ante las denominadas historias in medias res; si lo que se omite es la situación de equilibrio final, estamos ante una historia de final abierto. Para algunos autores, la decisión de omitir la situación inicial es decisiva desde el punto de vista político. Así, Molly Andrews (2007), señala cómo cambia significativamente la narrativa política posterior a los atentados del 11 de septiembre si se decide comenzar la historia por los atentados o por la política estadounidense previa.

En el caso de una campaña electoral, puede considerarse que la historia comienza in medias res, pues el conflicto principal es anterior al inicio de la campaña, y su final es abierto, pues, su narrativa es anterior al clímax y al desenlace de la historia. 
Algunos autores han identificado la estructura de historia mínima en relación con movimientos políticos, como el Tea Party estadounidense (Coughlin, 2011), o el storytelling de Ronald Reagan en las elecciones presidenciales de 1980 y 1984 y durante sus mandatos (Hanska, 2012).

\section{Los personajes en el storytelling político}

Desde una perspectiva narratológica, los personajes son los actores antropomorfos de una historia, siendo estos actores aquellas figuras narrativas que causan o experimentan los acontecimientos de la trama (Bal, 1985).

En el estudio de las campañas políticas, los personajes han sido estudiados, entre otras perspectivas, desde el punto de los arquetipos (Simorangkir y Pamungkas, 2017); aplicando análisis de contenido lingüísticos (Casañ-Pitarch, 2018; Gomez-Zara, Boon y Birnbaum, 2018); centrándose en una categoría de personaje (Anker, 2005; McHale, 2017); o en dos categorías (Klein y Mason, 2016; Alexander, 2010).

Actualmente, una aproximación frecuente es centrarse en tres categorías de personajes. Es el caso de Nimmo (1985), quien distingue entre héroe, villano y tonto (fool); y el del NPF (Shanahan, McBeth y Hathaway, 2011), que emplea las categorías de héroe (el personaje que potencialmente soluciona un problema político), villano (el que causa un problema) y victima (los dañados por el problema).

Más recientemente, se ha desarrollado una tipología de personajes específica para el análisis político, la denominada Teoría de los Personajes (Character Theory, CT) (Bergstrand y Jasper, 2018; Jasper, Young y Zuern, 2020), basada en las dimensiones EPA (Evaluación, Potencia y Actividad) del modelo de "significado afectivo" (affective meaning) propuesto por Osgood, Suci y Tannebaum (1957), que trata de explicar qué factores influyen en cómo los seres humanos reaccionamos a personas y cosas de nuestro entorno. Estas tres dimensiones han sido posteriormente utilizadas por la Teoría del Control del Afecto (Affect Control Theory, ACT) (Heise, 2007).

Así, la CT aplica dos criterios principales de las dimensiones EPA: la Moral (Morality), equivalente a la Evaluación; y la Potencia (Potency). Según su moral, los personajes pueden ser buenos o malos; según su potencia, fuertes o débiles. De esta forma, se llega a cuatro tipos de personajes principales: Héroe (fuerte, bueno); Villano (fuerte, malo); Víctima (débil, bueno/neutro); y Esbirro (débil, malo).

De los cuatro, por su capacidad para generar emociones que pueden influir en la acción política, estos autores destacan como más importantes al héroe, al villano y 
a la víctima. Así, el héroe se asocia a la esperanza; el villano, al miedo; y, la víctima, a la ira y a la compasión (Jasper, Young y Zuern, 2020).

Respecto a la Actividad, estos autores refieren que, aunque cualquiera de los personajes pueden encontrarse en un estado activo o pasivo, por lo general, el villano y sus esbirros están activos; las víctimas son personajes pasivos; y, el héroe, suele pasar de un estado de pasividad a uno de actividad, una vez que el villano ha actuado.

Por último, otro marco teórico utilizado en estudios sobre campañas políticas y relacionado con los personajes del storytelling es el que distingue entre la negatividad o positividad (negative campaigning/positive campaigning) de una campaña. Así, una campaña negativa - definida como "una crítica explícita al oponente político" (Hansen y Pedersen, 2008: 408) - caracteriza al adversario político como lo que la CT denomina villano; y, una campaña positiva, se centra en caracterizar al protagonista de la campaña como lo que la CT denomina héroe. Esta última perspectiva ha sido la más utilizada en relación con la narrativa en las investigaciones sobre las elecciones presidenciales brasileñas: las de desde 1989 hasta 2014 (Borba, 2015), y las de 2002 (Lourenço, 2009). Aunque tanto Borba como Lourenço señalan la escasez de estudios al respecto en el contexto brasileño. Por otro lado, esta perspectiva de estudio muestra que la posición en las encuestas de intención de voto es uno de los factores que más influyen en que un candidato emplee una campaña negativa: es menos probable que un candidato adopte una campaña negativa cuando va por delante en las encuestas que cuando va detrás o cuando las encuestas no dan un claro favorito (Damore, 2002; Hassell, 2019; Nai y Sciarini, 2015). En el caso de Portugal, Pereira (2019) observó este efecto en las campañas electorales legislativas de 2009 y 2011.

Entre los estudios sobre storytelling político que cuantifican las categorías que emplean, destaca el análisis de Clément, Lindemann y Sangar (2016) sobre los discursos relacionados con el uso de la fuerza en el contexto de la invasión de Irak en 2003, por parte de políticos de uno y otro bando. Estos autores aplican el marco de una narrativa que denominan de "héroe-protector", caracterizada por "estimular en el público objetivo ciertas emociones cuya función es convertir el uso de la fuerza en [...] una acción ineludible para preservar la autoestima [de los ciudadanos]" (Clément, Lindemann y Sangar, 2016: 998). Identifican cuatro personajes: héroe, agresor, víctima y cobarde; y cinco puntos de la trama: agresión actual, reacción a la agresión actual, agresión pasada, reacción a la agresión pasada y futuro estado final. Sus resultados muestran que los discursos que apoyan el uso de la fuerza recurren más a la figura del agresor que los discursos que no apoyan el uso de la fuerza; y que el recurso al héroe se asocia al recurso a las reacciones presentes y pasadas; 
el uso del agresor está relacionado con las agresiones presentes y pasadas; la víctima, con las agresiones presentes y el futuro estado final; y el cobarde aparece por igual en todos los puntos de la trama. Algunas de las categorías empleadas por Clément, Lindemann y Sangar pueden ser asociadas a las categorías narratológicas, como es el caso del héroe; del agresor, que puede ser considerado el villano; las agresiones, que pueden ser entendidas como los conflictos; y el futuro estado final, que puede ser considerado como la situación de equilibrio final.

\section{Objetivos e hipótesis}

El objetivo general de este estudio es analizar el storytelling de MRS y ASN en la campaña presidencial portuguesa de 2016. Los objetivos específicos han sido investigar la trama y los personajes en dichas narrativas y las relaciones entre ambas. Además, se ha planteado una hipótesis y una pregunta de investigación.

En relación con los personajes, se plantea la siguiente hipótesis:

Hipótesis 1: el candidato que iba por delante en las encuestas hizo menos alusiones al personaje del villano que el candidato que iba por detrás en las encuestas.

Respecto a la relación entra trama y personajes, hemos tomado como referencia el estudio de Clément, Lindeman y Sangar (2016), en el que algunas categorías pueden ser traspuestas a la estructura de historia mínima. En este sentido, creemos que no es pertinente formular una hipótesis, pero sí una pregunta de investigación que pudiera identificar un paralelismo que podría inducir a considerar una relación más general entre trama y personajes.

PR1:¿Recurrió más a los puntos de la trama de conflictos (Tr2 y $\operatorname{Tr} 3$ ) el candidato que más recurrió al personaje del villano?

\section{Metodología}

La metodología empleada en este estudio es de tipo mixta cualitativa-cuantitativa (Teddlie y Tashikkori, 2009), en la que se han analizado cuantitativamente los datos obtenidos a partir de los análisis de contenido de las muestras. Además, se han revisado las encuestas de intención de voto realizadas por Eurosondagem, Aximage y Universidad Católica desde noviembre de 2015 a enero de 2016.

El análisis de contenido se ha realizado en dos tipos de muestras: 1) los discursos de presentación de candidatura, pronunciados el 29 de abril de 2015 (ASN) y el 9 
de octubre de 2015 (MRS); y 2) las noticias relativas a las campañas de estos candidatos en dos periódicos portugueses de información general - el diario Público y el semanario Expresso -, en el periodo comprendido entre el 24 de noviembre de 2015 y el 23 de enero de 2016. En ambos periódicos se ha acotado el campo a artículos informativos (se han excluido los de opinión) de tres tipos:

i. aquellos que tratan en exclusiva sobre uno de los candidatos;

ii. entrevistas en exclusiva; y

iii. artículos sobre el debate entre ambos candidatos.

En relación con las noticias de los periódicos, se optó por analizar una fuente secundaria en lugar de una fuente primaria, las transcripciones completas de las declaraciones originales, por la imposibilidad de acceder a estas, ya que MRS utilizó a menudo notas personales a partir de las cuales improvisaba sus discursos y declaraciones.

La elección de Expresso y Público está motivada porque, en la prensa escrita portuguesa, estos dos periódicos destacan como "jornais de referência mais ligados à elite política e considerados importantes instrumentos na formação da opinião" (Miguel, Biroli y Salgado, 2017), y son los que mayor cobertura hacen de los partidos políticos portugueses (Graça, 2017: 61).

El semanario Expresso se publica los sábados pero, en diciembre de 2015, se publicó dos veces en jueves, por causa de las fiestas navideñas; y el semanario del 23 de enero se adelantó al viernes 22 debido a la jornada electoral. De este modo, los ejemplares de Expresso analizados han sido los correspondientes a las siguientes fechas: 28 de noviembre de 2015; 5, 12, 19, 24 y 31 de diciembre de 2015; 2, 9, 16 y 22 de enero de 2016.

Respecto al modo de análisis, tanto en los discursos de presentación de candidatura como en las informaciones de prensa escrita se han analizado los puntos de la trama y los personajes.

Para el estudio de la trama, según se ve en la Figura 2, se ha hecho una trasposición de la estructura de historia mínima a la campaña electoral del siguiente modo: (Tr1) Situación de Equilibrio Inicial, que refleja menciones a una situación anterior al conflicto principal de la campaña, o incluso a un pasado de mayor grandeza del país mucho más lejano, de las últimas décadas o incluso de los últimos siglos ("nossa capacidade de recuperar de um atraso de décadas, de séculos", ASN, Público, 16 de enero, p. 8); (Tr2) Conflictos previos al 9 de octubre de 2015 - cuando MRS anuncia 
5. El caso Banif hace referencia a la liquidación del Banco Internacional do Funchal (Banif), en diciembre de 2015, que supuso un gasto para el estado portugués de unos 3.000 millones de euros. su candidatura, después de haberlo hecho, meses atrás, ASN -, entre los que se ha considerado como conflicto principal la crisis económica y las políticas de austeridad implementadas por el gobierno del PSD para paliarla; ( $\operatorname{Tr} 3)$ Conflictos entre el 9 de octubre de 2015 y el 23 de enero de 2016, como la muerte de un joven en un hospital por falta de atención médica o las nuevas informaciones surgidas durante el periodo de campaña sobre el caso Banif5; (Tr4) Clímax, día de las elecciones, 24 de enero de 2016; y (Tr5) Situación de Equilibrio Final (promesas de los candidatos sobre qué harían si fueran elegidos presidente).

\section{FIGURA 2}

ESTRUCTURA DE TRAMA DE LA HISTORIA MÍNIMA APLICADA A LA CAMPAÑA ELECTORAL.

TRAMA EN CAMPAÑA ELECTORAL

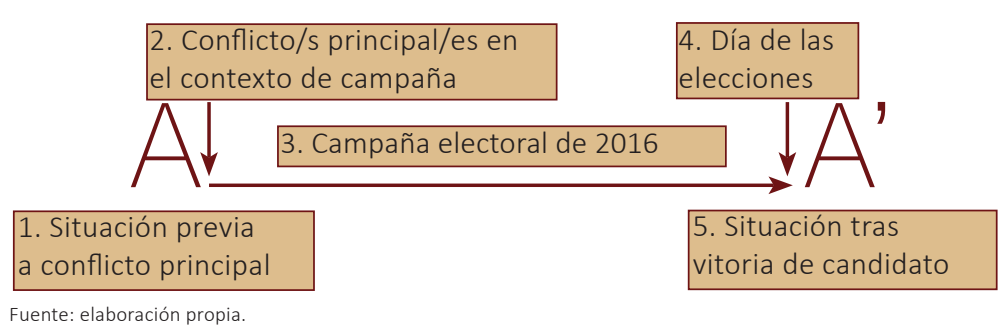

En cuanto a los personajes, se han analizado la caracterización del héroe, del villano y de las víctimas. En relación con el héroe, se han analizado las dimensiones de moralidad, potencia y actividad; en el caso del villano, dado que todas las alusiones han estado relacionadas con la moralidad, se ha distinguido entre villano específico y villano inespecífico. En la categoría de villano específico se ha incluido todas aquellas menciones por parte de los candidatos a personas o entidades concretas, con su nombre. Por ejemplo, cuando un candidato menciona al otro aludiendo a una característica suya negativa. O "Cavaco Silva não protegeu as pessoas" (ASN, Expresso, 9 de enero, p. 7). En la categoría de villano inespecífico se ha incluido las alusiones de un candidato a un villano al que no se nombre explícitamente. Por ejemplo: "A classe política está habituada a campanhas de tal forma dispendiosas e estruturadas que depois é difícil encolher" (MRS, Expresso, 12 de diciembre, p. 6).

Los datos se han analizado de dos formas. En primer lugar, de forma separada para, por un lado, los discursos de presentación; y, por otro, para las informaciones de prensa escrita. En segundo lugar, se ha realizado un análisis de conglomerados o clusters mediante la distancia de Ward empleando seis unidades: MRS-Público, MRS-Expresso; MRS-Discurso de presentación de candidatura, ASN-Público, ASN-Expresso; y ASN-Discurso de presentación de candidatura. Este instrumento estadístico sirve para establecer relaciones entre estas seis unidades, más allá del candidato y de si pertenece a un discurso o a las noticias de uno u otro periódico. 


\section{Resultados}

En primer lugar, se presentan los resultados por separado para prensa escrita y para los discursos de presentación de candidatura. Primero, en relación con la trama y, luego, en relación con los personajes. A continuación, se presenta el análisis de conglomerados y, finalmente, se hace una revisión de las encuestas de intención de voto.

\section{La trama}

Ambos candidatos recurren de forma muy similar a los puntos de la trama: 55 alusiones (MRS) y 52 alusiones (ASN). Los porcentajes para cada punto también muy similares, como muestra el Gráfico 1 . Destacan las alusiones a $\operatorname{Tr} 5$, que refleja las menciones a lo que harán los candidatos una vez ganen las elecciones, y que representan alrededor de la mitad del total de alusiones a puntos de la trama. Asimismo, destacan también las alusiones a Tr4, es decir, a la jornada electoral, que refleja las menciones a la petición del voto para el candidato o, en general, a que se acuda a votar. Esta elevada alusión a la jornada electoral podría tener distintos significados según el candidato. MRS pide que se acuda a votar, sin especificar a qué candidato, ya que una alta abstención podría hacerle correr el riesgo de una segunda vuelta, en la que podría repetirse lo sucedido en 1986, cuando Diogo Pinto de Freitas do Amaral, del CDS, obtuvo la victoria en la primera vuelta sobre el socialista Mario Soares, pero no por mayoría absoluta, y en la segunda vuelta Mario Soares resultó victorioso.

Por su parte, en ASN, las alusiones a la jornada electoral hacen referencia a la esperanza en una segunda vuelta ("nada ficará decidido no domingo" (Expresso, 22 de enero de 2016). Esta esperanza se veía reforzada por el descenso significativo de Maria de Bélem en las dos encuestas previas a la jornada electoral.

GRÁFICO 1

\section{PUNTOS DE LA TRAMA EN PRENSA ESCRITA}

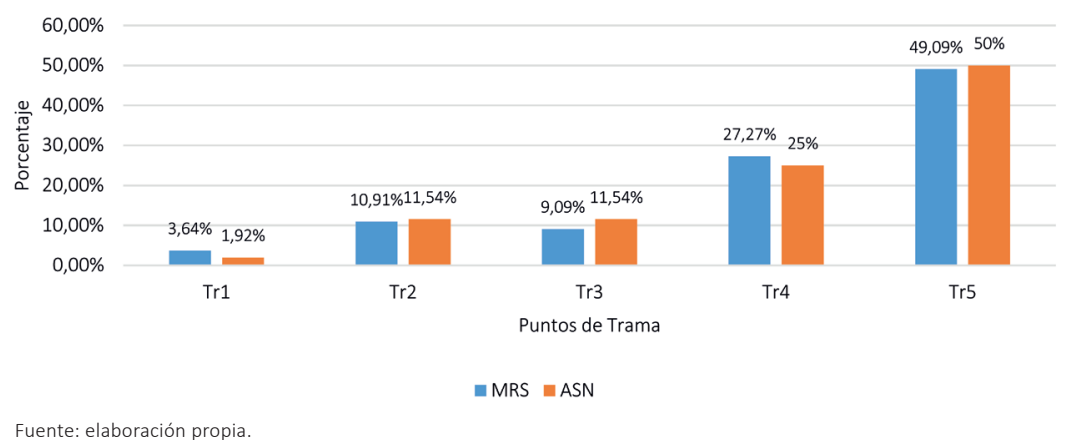


En los discursos de presentación de candidatura se observa una gran diferencia entre ambos candidatos en cuanto al número de alusiones. MRS solo hace referencia a ellos en 3 ocasiones, dos de ellas a Tr2 (conflicto principal de las elecciones) y una vez a Tr1 (situación inicial, previa a la campaña electoral). Por su parte, ASN hace 37 alusiones a puntos de la trama, 4 de cada 5 de ellas, a $\operatorname{Tr} 5$ (situación de equilibrio final). En este sentido, en el caso de MRS, la conclusión que puede extraerse es que apenas basa su discurso en la trama, aunque, cuando lo hace, alude a lo que va a enfrentarse y a las consecuencias de su victoria. En el caso de ASN, en cambio, sí es significativa la alusión a la trama en su discurso, y es coherente con lo que manifiesta en prensa escrita.

TABLA 2

PUNTOS DE LA TRAMA EN DISCURSOS

DE PRESENTACIÓN DE CANDIDATURA

\begin{tabular}{|c|c|c|c|c|c|}
\cline { 2 - 6 } \multicolumn{1}{c|}{} & TR1 & TR2 & TR3 & TR4 & TR5 \\
\hline MRS & $33,33 \%$ & $66,67 \%$ & $0,00 \%$ & $0,00 \%$ & $0,00 \%$ \\
\hline ASN & $0,00 \%$ & $10,81 \%$ & $5,41 \%$ & $2,70 \%$ & $81,08 \%$ \\
\hline
\end{tabular}

Los personajes

Ambos candidatos hicieron 65 alusiones a personajes (héroe, villano o víctima) en la prensa escrita analizada:. Para ambos candidatos, el periódico Público es el que más incluye las alusiones de los candidatos a personajes:40, en el caso de MRS; y 54 , en el caso de SN.

En la prensa escrita estudiada, según muestra el Gráfico 2, en ambos candidatos es más importante la caracterización del héroe que la del villano, pero MRS menciona casi seis veces más la figura narrativa del héroe que la del villano, mientras que en ASN destaca el elevado porcentaje de alusiones al villano.

Respecto a las víctimas, como se aprecia en el Gráfico 2, en ambos candidatos los porcentajes son muy bajos.

En cuanto a las dimensiones del héroe, como se ve en el Gráfico 3, los porcentajes son similares en ambos candidatos. Destacan las alusiones a la dimensión moral del héroe, casi nueve de cada diez alusiones en ambos candidatos.

Así, mientras que el storytelling de MRS mantiene una preeminencia de la figura del héroe -con un mensaje que puede ser resumirse como "fazer pontes, não só 
GRÁFICO 2

LOS PERSONAJES EN PRENSA ESCRITA

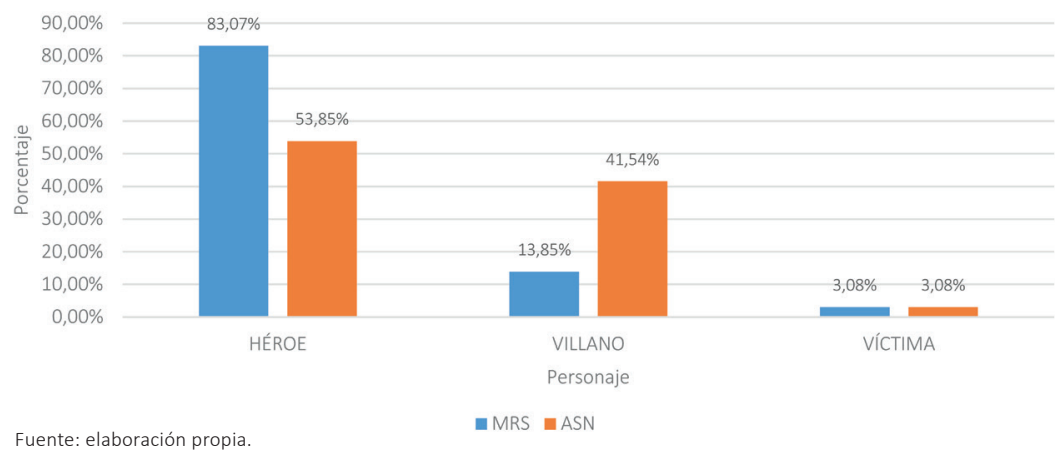

GRÁFICO 3

DIMENSIONES DEL HÉROE

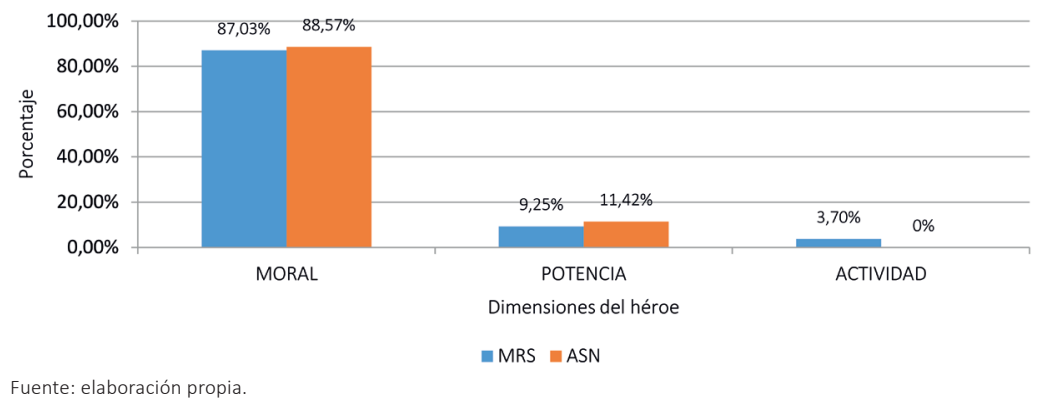

entre o Governo, mas também entre a oposição" (Público, 17 de diciembre, p. 12), defendiendo la moderación y el consenso-, ASN, si bien la mitad de sus alusiones son al héroe, en cuatro de cada diez veces se refiere al villano, principalmente, a MRS (representante de "uma visão das políticas do antigamente" (Público, 10 de diciembre de 2015, p. 6), "vaidoso", "só gostar de se ouvir a si próprio", "auto-suficiente e sem ouvir ninguém" (Público, 5 de enero de 2016, p. 6), es "responsável das fracturas que marcaram o país" (Público, 8 de nero de 2015, p. 6), "uma das figuras do regime" (Público, 10 de enero de 2015, p. 7), o "quem vê a política como um clube" (Público, 11 de enero de 2016, p. 2), entre otras.

Uno de los principales ataques de ASN a MRS a lo largo de toda la campaña fue que quiere contentar a todos, ya que formó parte del gobierno responsable de la crisis y de las políticas de austeridad que ha sufrido la ciudadanía, pero pretende defender una posición de consenso con el gobierno socialista de António Costa. A ello reacciona MRS, especialmente en torno al debate televisivo mantenido entre 
ambos candidatos el 7 de enero de 2016 en la cadena SIC, acusando a ASN de inexperiencia política y de querer "ir de soldado raso a general" (Público, 8 de enero de 2016, p. 6).

En relación con la confianza "heroica" en la victoria por parte de MRS, cabe resaltar su declaración, casi un mes antes de la jornada electoral, de que "daqui a semanas sou Presidente da República" (Expresso, 12 de diciembre de 2015, Portada).

En la categoría de villano, hemos distinguido entre las menciones a un villano específico o a un villano inespecífico. En el Gráfico 4 se observa cómo la mayoría de las veces que ASN utiliza la categoría de villano, lo hace aludiendo a un villano específico. Por su parte MRS recurre a la categoría del villano específico menos de la mitad de las veces que ASN. En el caso de ASN, el villano es, principalmente, MRS, de quien critica su relación al anterior presidente, Cavaco Silva (PSD), que implantó las medidas de austeridad para combatir la crisis, o su "duplicidad" de posiciones como comentador político y candidato, así como el hecho de que MRS "dizem uma coisa e o seu contrário" y muestra una "falta de clareza" que, en su opinión, constituye "um problema" (Público, 7 de enero de 2016, p.15).

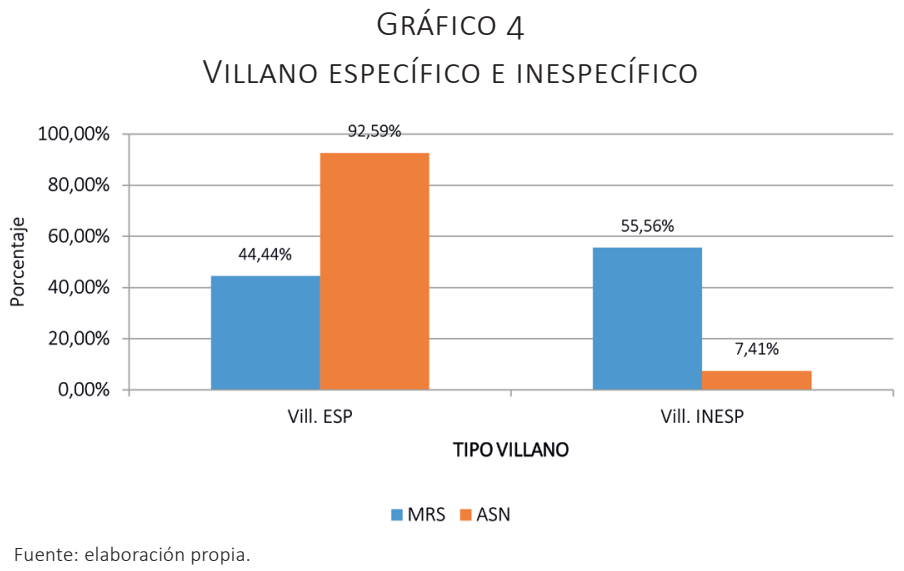

En los personajes presentes en los discursos de presentación de candidatura, como se observa en la Tabla 3, predomina significativamente la presencia del héroe. En el caso de MRS, representa el $100 \%$ de las alusiones a personajes. Es, además, notable la ausencia, en ambos candidatos, de referencias al villano. Por último, ASN hace referencia a las víctimas en un $15,79 \%$ de sus alusiones a cualquier personaje.

La posible razón de estas diferencias es que MRS hace un recorrido por su biografía, destacando sus virtudes morales, su fortaleza y su sentido de sacrificio por los 
TABLA 3

LOS PERSONAJES EN LOS DISCURSOS

DE PRESENTACIÓN DE CANDIDATURA

\begin{tabular}{|c|c|c|c|}
\cline { 2 - 4 } \multicolumn{1}{c|}{} & HÉROE & VILLANO & VÍCTIMA \\
\hline MRS & $100,00 \%$ & $0,00 \%$ & $0,00 \%$ \\
\hline ASN & $84,21 \%$ & $0,00 \%$ & $15,79 \%$ \\
\hline
\end{tabular}

Fuente: elaboración propia.

votantes, ya que, de ser elegido, abandonaría su actividad académica y televisiva, mientras que, en el caso de ASN, aunque centra su discurso en su programa, lo que reflejan las alusiones a la situación de equilibrio final, alude asimismo a las víctimas en cerca de una de cada seis veces que hace referencia a un personaje, lo que apunta a una estrategia diferente de la de MRS.

\section{Análisis de conglomerados}

En el análisis de conglomerados o clusters se ha tratado de ver si existe relación entre las distintas categorías analizadas, más allá de que pertenezcan a uno u otro candidato o a distintos tipos de texto. Así, en la elaboración de estos conglomerados se han tenido en cuenta tanto los puntos de la trama ( $\operatorname{Tr} 1-\operatorname{Tr} 5)$, como los personajes y sus características (en el caso del héroe, desglosado en moral, actividad y potencia; y, en el caso del villano, desglosado en específico e inespecífico), como los diferentes textos (Público, Expresso y discursos de candidatura).

Mediante este análisis se observan tres conglomerados principales (ver Tablas 4, 5 y 6), lo que refleja una relación más estrecha entre los textos que pertenecen a cada uno de ellos.

TABLA 4

CONGLOMERADO 1

\begin{tabular}{|c|c|c|c|}
\hline Variable & $\begin{array}{c}\text { \% de la frecuencia } \\
\text { en la partición }\end{array}$ & $\begin{array}{c}\text { \% de la frecuencia } \\
\text { en la muestra }\end{array}$ & $\begin{array}{c}\text { \% de la partición } \\
\text { en la frecuencia }\end{array}$ \\
\hline Trama5 & 39,2 & 26,0 & 91,6 \\
\hline Trama2 & 7,7 & 5,6 & 83,3 \\
\hline \multicolumn{5}{|l}{} \\
\hline Heroe_potencia & 2,6 & 5,3 & 29,4 \\
\hline Villano_especifico & 3,6 & 9,1 & 24,1 \\
\hline Heroe_moral & 22,2 & 32,9 & 41,0 \\
\hline
\end{tabular}


TABLA 5

CONGLOMERADO 2

\begin{tabular}{|c|c|c|c|}
\hline Variable & $\begin{array}{c}\text { \% de la frecuencia } \\
\text { en la partición }\end{array}$ & $\begin{array}{c}\text { \% de la frecuencia } \\
\text { en la muestra }\end{array}$ & $\begin{array}{c}\text { \% de la partición } \\
\text { en la frecuencia }\end{array}$ \\
\hline Heroe_moral & 57,1 & 32,9 & 34,3 \\
\hline Heroe_potencia & 14,3 & 5,3 & 52,9 \\
\hline \multicolumn{4}{|c|}{} \\
\hline Villano_especifico & 0,0 & 9,1 & 0,0 \\
\hline Trama5 & 6,3 & 26,0 & 4,8 \\
\hline
\end{tabular}

TABLA 6

CONGLOMERADO 3

\begin{tabular}{|c|c|c|c|}
\hline Variable & $\begin{array}{c}\text { \% de la frecuencia } \\
\text { en la partición }\end{array}$ & $\begin{array}{c}\text { \% de la frecuencia } \\
\text { en la muestra }\end{array}$ & $\begin{array}{c}\text { \% de la partición } \\
\text { en la frecuencia }\end{array}$ \\
\hline Villano_especifico & 35,5 & 9,1 & 75,9 \\
\hline \multicolumn{4}{|c|}{} \\
\hline Trama2 & 0,0 & 5,6 & 0,0 \\
\hline Trama5 & 4,8 & 26,0 & 3,6 \\
\hline Fuente: elaboración propia.
\end{tabular}

El primer conglomerado (Tabla 4) está formado por el discurso de candidatura de ASN, los artículos en Expresso sobre ASN y los textos en Público sobre MRS. Se caracteriza por una mayor frecuencia - en los textos que incluye, y respecto al resto de los textos - de $\operatorname{Tr} 5$ y $\operatorname{Tr} 2: \operatorname{Tr} 5$ (\% de partición en la frecuencia = 91,6) y $\operatorname{Tr} 2$ (\% de partición en la frecuencia $=83,3$ ). Ello significa que, en todos los textos analizados para cualquier candidato y texto, el 91,6\% de las veces que se menciona $\operatorname{Tr} 5$ y el $83,3 \%$ de las alusiones a $\operatorname{Tr} 2$, se encuentran en las unidades pertenecientes a este conglomerado. Asimismo, los textos pertenecientes a este conglomerado se caracterizan por una menor frecuencia del villano específico (\% de partición en la frecuencia $=24,1$ ), del héroe en su cualidad de Potencia (\% de partición en la frecuencia $=29,4)$ y del héroe en su cualidad Moral (\% de partición en la frecuencia $=41$ ).

El segundo conglomerado (Tabla 5) está formado por el discurso de candidatura de MRS y los artículos en Expresso sobre MRS. Se observa en él una mayor frecuencia de la moral del héroe y de la potencia del héroe; y una menor frecuencia del villano específico y de $\operatorname{Tr} 5$.

Por último, el tercer conglomerado (Tabla 6) está formado por los artículos sobre ASN en Público, y se caracteriza por la mayor presencia del villano específico y por una menor frecuencia de $\operatorname{Tr} 2$ y $\operatorname{Tr} 5$. 
De forma general, podemos decir que el hallazgo más significativo derivado del análisis de conglomerados, tanto sobre los personajes como sobre la trama, es que Expresso y Público tratan de forma muy diferente estas referencias - ataques, principalmente, a MRS - por parte de ASN. Así, Expresso refleja estas referencias de forma muy reducida con respecto a Público, lo que hace que las noticias sobre ASN en cada diario aparezcan en conglomerados diferentes.

\section{Conclusiones}

El objetivo de este estudio ha sido investigar el storytelling político en una campaña presidencial, centrándose en la trama y los personajes, para así contribuir a la literatura académica en este campo.

Respecto a los personajes, los datos muestran que, en sus discursos de presentación de candidatura, ambos candidatos se centraron en caracterizarse como héroes, y ambos evitaron caracterizar como villanos a sus adversarios. Sin embargo, en el discurso de ASN ya está presente la principal diferencia que se observa en el análisis de la prensa escrita, es decir, una significativa mayor alusión al villano y, en concreto, a la categoría de villano específico.

De este modo, la hipótesis planteada se confirma, ya que el candidato que va por detrás en las encuestas hace un mayor uso del personaje del villano, es decir, emplea una campaña más negativa, que el candidato que va por delante. Esto es coherente con otros estudios (Damore, 2002; Hassell, 2019; Nai y Sciarini, 2015; Pereira, 2019). Esta caracterización negativa se ve intensificada por las alusiones al villano específico, en una de cada diez veces, en el caso de ASN.

En el análisis de conglomerados se aprecia que es en el diario Público donde el villano específico es más mencionado por ASN. Esta diferencia resulta sorprendente si consideramos que ninguno de los dos periódicos está adscrito a tendencias políticas relacionadas con alguno de los candidatos (Paquete de Oliveira, 2016). La explicación a esta diferencia sería oportuno investigarla en una futura investigación sobre el tono, más o menos moderado de ambos medios.

Respecto a la trama en prensa escrita, las narrativas son muy semejantes. Ambos hacen referencia a lo que harán después de ser elegidos ( $\operatorname{Tr} 5$ ) casi la mitad de las veces de las veces. Esta estrategia - la de hacer imaginar al consumidor potencial una satisfacción producida por la obtención del producto -, ha sido demostrada en otros ámbitos (Shiv y Huber, 2000). 
Así, en relación con la pregunta de investigación planteada respecto a los puntos de la trama, en prensa escrita no se observó que el candidato que hizo un mayor uso de la figura del villano aludiera significativamente más a los conflictos, lo que no permite establecer un paralelismo con los resultados de Clément, Lindemann y Sangar (2016). En este sentido, sería necesarias futuras investigaciones para esclarecer estas relaciones entre trama y personajes en el contexto de las campañas electorales.

Aunque la prensa escrita escogida como parte de la muestra de este estudio es competente y experimentada en el tratamiento de la información política, una limitación de este estudio es el no haber podido acceder a transcripciones completas de las declaraciones y discursos de los candidatos, lo que supone un filtro.

La importancia de este estudio es que emplea una aproximación a la trama del storytelling político desde la narratología aplicando la estructura de historia mínima, lo cual, aunque algunos autores habían hallado paralelismos con ella en algunas narrativas políticas (Coughlin, 2011; Hanska, 2012), según creen estos autores, no se había utilizado anteriormente en el análisis de contenido. Otro hallazgo interesante es que, en nuestro estudio, la narrativa de los discursos de presentación de candidatura evita las alusiones al villano, aunque ambos discursos sean muy diferentes. Asimismo, en prensa escrita, se observa que los puntos de la trama empleados son muy semejantes en los dos candidatos, y que el más utilizado es la situación de equilibrio final. Futuras investigaciones podrían tratar de esclarecer si esto frecuente en las campañas presidenciales. Finalmente, este estudio confirma la relación entre el uso de la figura del villano y la posición en las encuestas de los candidatos, que otros autores habían observado en el contexto electoral portugués y en otros contextos electorales.

\section{Referencias}

ALEXANDER, Jeffrey. The performance of politics. Obama's Victory and the democratic struggle for power. New York: Oxford University Press, 2010.

ANDREWS, Molly. Shaping history: narratives of political change. Cambridge (UK): Cambridge University Press, 2007.

ANKER, Elisabeth. Villains, victims and heroes: melodrama, media, and September 11. Journal of Communication, v. 55, n. 1, p. 22-37, 2005. 
ANSOLABEHERE, Stephen; IYENGAR, Shanto. Going negative. How political advertisements shrink \& polarize the electorate. New York: The Free Press, 1995.

ARISTÓTELES. Poética. México (DF): Bibliotheca Scriptorvm Graecorvm et Romanorvm Mexicana, 1946.

BAL, Mieke. Narratology. Introduction to the theory of narrative. Toronto (CA): University of Toronto Press, 1985.

BARTON, Jared; CASTILLO, Marco; PETRIE, Ragan. Negative campaigning, fundraising, and voter turnout: a field experiment. Journal of Economic Behavior \& Organization, v. 121, p. 99-113, 2016.

BERGSTRAND, Kelly; JASPER, James M. Villains, victims, and heroes in character theory and affect control theory. Social Psychology Quarterly, v. 81, n. 3, p. 228247, 2018.

BJÖRNINEN, Samuli; HATAVARA, Mari; MÄKELÄ, Maria. Narrative as social action: a narratological approach to story, discourse and positioning in political storytelling. Journal of Social Research Methodology, v. 23, n. 4, p. 437-449, 2020.

BORBA, Felipe. Propaganda negativa nas eleições presidenciais brasileiras. Opinião Pública, v. 21, n.2, p. 268-295, 2015.

BOTO, Carlota. António Nóvoa: uma vida para a educação. Educação e Pesquisa, v. 44, p. 1-24, 2018.

CASAÑ-PITARCH, Ricardo. Mr. president, discourse matters: a contrastive analysis of Donald Trump and Barack Obama's siscourse. RUDN Journal of Language Studies, Semiotics and Semantics, v. 9, n. 1, p. 173-185, 2018.

CASTROMIL, Anton R. Negativismo mediático y campaña electoral en las elecciones generales de 2008. REIS, v. 139, p. 163-174, 2012.

CHATMAN, Seymour. Story and discourse. Narrative structure in fiction and film. Ithaca (NY): Cornell University Press, 1978.

CHARTERISBLACK, Jonathan. Analysing political speeches: rhetoric, discourse and metaphor. Hampshire (UK): Palgrave Macmillan, 2014.

CHOU, Mark; BLEIKER, Roland; PREMARATNA, Nilanjana. Elections as theater. Washington (DC): American Political Science Association, 2016. 
CLÉMENT, Maéva; LINDEMANN, Thomas; SANGAR, Eric. The "hero-protector narrative": manufacturing emotional consent for the use of force. Political Psychology, v. 38, n. 6, p. 991-1008, 2016.

COUGHLIN, Alex T. The challenges of populism: an analysis of teaparty structuring narratives. Tesis Doctoral, Colorado State Univesrity, 2011.

CURINI, Luigi. The direction of Valence campaigning in two dimensions. In:

(Org.). Corruption, ideology, and populism. London: Palgrave Macmillan, 2017.

DAMORE, David F. Candidate strategy and the decision to go negative. Political Research Quarterly, v. 55, n. 3, p. 669-686, 2002.

DEIGHTON, John, ROMER, Daniel; MCQUEEN, Josh. Using drama to persuade. Journal of Consumer Research, v. 16, n. 3, p. 335, 1989.

FIGUEIRAS, Rita. O efeito Marcelo: o comentário político na televisão. Lisboa: Fundação Francisco Manuel dos Santos, 2019.

GOMEZ-ZARA, Diego; BOON, Miriam; BIRNBAUM, Larry. Who is the hero, the villain, and the victim?: detection of roles in news articles using natural language techniques. IUI '18: 23rd International Conference on Intelligent User Interfaces, p. 311-315, March 2018.

GRAÇA, Francisco. A política e os media: o enviesamento da imprensa portuguesa em 2009 e 2015. Dissertação de mestrado. Instituto Universitário de Lisboa (ISCTE/ IUL), 2017.

GRAY, Garry; JONES, Michael D. A qualitative narrative policy framework? Examining the policy narratives of US campaign finance regulatory reform. Public Policy and Administration, v. 31, n. 3, p. 193-220, 2016.

HANSEN, Kasper M.; PEDERSEN, Rasmus T. Negative campaigning in a multiparty system. Scandinavian Political Studies, v. 31, n. 4, p. 408-427, 2008.

HANSKA, Jan. Reagan's mythical America. Storytelling as political leadership. New York: Palgrave Macmillan, 2012.

HASSELL, Hans J. G. Desperate times call for desperate measures: electoral competitiveness, poll position, and campaign negativity. Political Behavior, p. 1-23, Aug. 2019.

HEISE, David R. Expressive order. Boston (MA): Springer, 2007. 
JASPER, James M.; YOUNG, Michael P.; ZUERN, E. Public characters. The politics of reputation and blame. New York: Oxford University Press, 2020.

. Character work in social movements. Theory and Society, v. 47, n. 1, p. 113131, 2018.

KIRKPATRICK, Kellee J.; STOUTENBOROUGH, James W. Strategy, narratives, and reading the public: developing a micro-level theory of political strategies within the narrative policy framework. Policy Studies Journal, v. 46, n. 4, p. 949-977, 2018.

KLEIN, Sarah; MASON, Tom. How to win an election. The New York Times. 18 Feb. 2016. Recuperado de: <https://www.nytimes.com/2016/02/18/opinion/how-towin-an-election.html>. Fecha de acceso: 05 Jun. 2020.

LAU, Richard R., SIGELMAN, Lee; ROVNER, Ivy B. The effects of negative political campaigns: a meta-analytic reassessment. Journal of Politics, v. 69, n. 4, . 11761209, 2007.

LOURENÇO, Luiz Claudio. Propaganda negativa: ataque versus votos nas eleições presidenciais de 2002. Opinião Pública, v. 15, n. 1, p. 133-158, 2009.

MCBATH, James H.; FISHER, Walter R. Persuasion in presidential campaign communication. Quarterly Journal of Speech, v. 55, n. 1, p. 17-25, 1969.

MCBETH, Mark K.; SHANAHAN, Elizabeth A. Public opinion for sale: the role of policy marketers in greater yellowstone policy conflict. Policy Sciences, v. 37, n. 3, p. 319-338, 2004.

MCBETH, Mark K.; SHANAHAN, Elizabeth A.; JONES, Michael D. The science of Storytelling: measuring policy beliefs in Greater Yellowstone. Society \& Natural Resources, v. 18, n. 5, p. 413-442, 2005.

MCHALE, John P. Media coverage of corruption and scandal in the 2016 presidential election: fantasy themes of crooked Hillary and corrupt businessman Trump. In: LIND, N. S.; ROBE-HEMP, C. E. (Orgs.). Corruption, Accountability and Discretion, v. 29, p. 107-123, 2017.

MCMORRIS, Claire; ZANOCCO, Chad; JONES, Michael. Policy narratives and policy outcomes: an NPF examination of Oregon's Ballot Measure 97. Policy Studies Journal, v. 46, n. 4, p. 771-797, 2018.

MERELMAN, Richard M. The dramaturgy of politics. The Sociological Quarterly, v. 10, n. 2, p. 216-241, 1969. 
MIGUEL, Luis Felipe; BIROLI, Flávia; SALGADO, Susana. Meios de comunicação e política: evolução histórica e comparações entre Brasil e Portugal. In: PRIOR, H.; GUAZINA, L.; ARAUJO, B. (Orgs.). Diálogos lusófonos em comunicação e política. Lisboa: LabCom Books, 2017.

NAI, Alessandro; SCIARINI, Pascal. Why "going negative?" Strategic and situational determinants of personal attacks in Swiss direct democratic votes. Journal of Political Marketing, v. 17, n. 4, p. 1-31, 2015.

NIMMO, Dan. Elections as ritual drama. Society, v. 22, n. 4, p. 31-38, 1985.

OSGOOD, Charles E.; SUCl, George J.; TANNEBAUM, Percy H. The measurement of meaning. Urbana (IL): The University of Illinois Press, 1957.

PAGE, Janis T.; DUFFY, Margaret E. What does credibility look like? Tweets and walls in U.S. presidential candidates' visual storytelling. Journal of Political Marketing, $\mathrm{v}$. 17, n. 1, p. 3-31, 2016.

PAQUETE DE OLIVEIRA, José Manuel. Não há jornais de direita nem de esquerda em Portugal. Público, 06 Jun. 2016. Recuperado de: <ttps://www.publico. pt/2016/06/06/sociedade/opiniao/nao-ha-jornais-de-direita-nem-de-esquerdaem-portugal-1734159>. Fecha de acceso: 11 Jun. 2020.

PEREIRA, Miguel M. Do parties respond strategically to opinion polls? Evidence from campaign statements. Electoral Studies, v. 59, p. 78-86, 2019.

As eleições presidenciais através das sondagens, 12 Jan. 2016. Recuperado de: <https://miguelmpereira.com/2016/01/12/as-eleicoes-presidenciais-atraves-das-sondagens/>. Fecha de acceso: 08 Jun. 2020.

POLKINGHORNE, Donald E. Narrative knowing and the human sciences. Albany (NY): State University of New York Press, 1988.

POLLETTA, Francesca. It was like a fever. Chicago (IL): University of Chicago Press, 2006.

SHANAHAN, Elizabeth A., MCBETH, Mark K. y HATHAWAY, Paul. L. Narrative policy framework: the influence of media policy narratives on public opinion. Politics \& Policy, v. 39, n. 3, p. 373-400, 2011.

SIMORANGKIR, Deborah N.; PAMUNGKAS, Sigit. Strategic political communication through storytelling: A case study of the "Democreative Tales of Jokowi's Blusukan" comics. Public Relations Journal (Special Issue), p. 1-22, 2017. 
STONE, Deborah. Policy paradox: the art of political decision making. New York: WW. Norton, 2002.

SHIV, Baba; HUBER, Joel. The impact of anticipating satisfaction on consumer choice. Journal of Consumer Research, v. 27, n. 2, p. 202-216, 2000.

SWERTS, Thomas. Gaining a voice: storytelling and undocumented youth activism in Chicago. Mobilization: An International Quarterly, v. 20, n. 3, p. 345-360, 2015.

TEDDLIE, Charles; TASHAKKORI, Abbas. Foundations of mixed methods research. Los Angeles (CA): Sage, 2009.

TODOROV, Tzvetan. Literatura y significación. Barcelona (ES): Planeta, 1963.

VROMEN, Ariadne; COLEMAN, William. Online campaigning organizations and storytelling strategies: GetUp! in Australia. Policy \& Internet, v. 5, n. 1, 76-100, 2013.

WOLF, Larissa. The use of rhetorical devices in selected speeches by Hillary Clinton and Donald Trump during the electoral campaign 2016. Disertación de Máster, Universidad de Viena, 2017. 